\title{
On Fuzzy Measure on Fuzzy sets
}

\author{
Noori F. Al-Mayahi \\ Department of Mathematics \\ College ofComputer Science and IT \\ University of Al-Qadisiya \\ Email: nfam60@yahoo.com
}

\author{
Karrar S. Hamzah \\ Department of Mathemat \\ College of Computer Science and IT \\ University of Al-Qadisiya \\ Email: karrar638@gmail.com
}

Recived : 2\4\2017

Revised : 14\5\2017

Accepted : 29\5\2017

\begin{abstract}
In this paper, we study the fuzzy measure on fuzzy sets and prove some new properties.
Keywords: Fuzzy measure, semi continuous fuzzy measure, null additive fuzzy measure, fuzzy measure accountably weakly null-additive fuzzy measure.
\end{abstract}

Mathematics subject classification : 20CXX .

\section{Introduction}

The fuzzy measure, defined on $\sigma$-field, was introduced by Sugeno [4]. Ralescu and Adams [10] generalized the concepts of fuzzy measure and fuzzy integral to the case that the value of a fuzzy measure can be infinite, and to realize an approach from Subjective.

Wang [7,11] and Kruse [17] studied some structural characteristics of fuzzy measures and proved several theorem about fuzzy measure.

Wang $[7,11]$ introduced the concept of 'autocontinuity of a set function', used it with regard to the above-mentioned researches, and obtained a series of new results.

The notion of fuzzy measure was extended by Avallone and Barbieri, Jiang and Suzuki [14] Narukawa and Murofushi [8] , Ralescu and Adams [10] as a set function which was defined on $\sigma$-field with values in $[0, \infty]$. After that, many authors studied the fuzzy measure and proved some results about it as Guo and Zhang [8] Kui [13], Li and Yasuda [6] Lushu and Zhaohu [15] Minghu [16].

In this paper, we mention the definition of Fuzzy Measure on Fuzzy

Set with some Properties, and prove some new relations deal with fuzzy measure.

\section{Definition (1): [18, 19]}

Let $\Omega$ be anon empty set, a fuzzy set $A$ in $\Omega$ (or a fuzzy subset in $\Omega$ ) is a function from $\Omega$ into $I$, i.e. $\mathrm{A} \in \mathrm{I}^{\Omega} . A(x)$ is interpreted as the degree of membership of element $x$ in a fuzzy set $A$ for each $x \in \Omega$. a fuzzy set $A$ in $\Omega$ is can be represented by the set of pairs:

$$
A=\{(x, A(x)): x \in \Omega\}
$$

Note that every ordinary set is fuzzy set, i.e. $\mathrm{P}(\Omega) \subseteq I^{\Omega}$.

Definition (2): [1, 2]

A family $\mathcal{F}$ of fuzzy sets in a set $\Omega$ is called a fuzzy $\sigma$-field on a set $\Omega$ If,

1. $\phi, \Omega \in \mathcal{F}$.

2. If $A \in \mathcal{F}$, then $A^{c} \in \mathcal{F}$.

3. If $\left\{A_{n}\right\} \subset \mathcal{F}, n=1,2,3, \ldots, \quad$ then $\bigcup_{n=1}^{\infty} A_{n} \in \mathcal{F}$.

Evidently, an arbitrary $\sigma$-field must be fuzzy $\sigma$-field.

A fuzzy measurable Space is a $\operatorname{pair}(\Omega, \mathcal{F})$, where $\Omega$ is a set and $\mathcal{F}$ is a fuzzy $\sigma$-field on $\Omega$. a fuzzy set $A$ in $\Omega$ is called fuzzy measurable (fuzzy measurable with respect to the fuzzy $\sigma$-field ) if $A \in \mathcal{F}$, i.e. any member of $\mathcal{F}$ is called a fuzzy measurable set.

Definition (3) [3]:

Let $(\Omega, \mathcal{F})$ be a fuzzy measurable space. A set function $\mu: \mathcal{F} \rightarrow[0, \infty]$ is said to be

(1) Finite if, $\mu(A)<\infty$ for each $A \in \mathcal{F}$.

(2) Semi-finite, if for each $A \in \mathcal{F}$ with $\mu(A)=\infty$, there exists $B \in \mathcal{F}$ with $B \subseteq A$ and $0<\mu(B)<\infty$. 
(3)

Bounded, if

$$
\sup \{|\mu(A): A \in \mathcal{F}|\}<\infty
$$

(4) $\sigma$-finite, if for each $A \in \mathcal{F}$, there is a sequence $\left\{A_{n}\right\}$ of sets in $\mathcal{F}$ such that

$$
A \subset \bigcup_{n=1}^{\infty} A_{n}
$$

And $\mu\left(A_{n}\right)<\infty$ for all $n$

(5) Additive if,

$\mu(A \cup B)=\mu(A)+\mu(B) \quad$ whenever $A, B \in \mathcal{F}$ and $\mathbf{A} \cap \mathbf{B}=\emptyset$.

(6)

Finitely additive if,

$$
\mu\left(\bigcup_{k=1}^{n} A_{k}\right)=\sum_{k=1}^{n} \mu\left(A_{k}\right)
$$

whenever $A_{1}, A_{2, \ldots}, A_{n}$ are disjoint sets in $\mathcal{F}$.

(7) $\sigma$-additive (sometimes called

Completely additive, or

A counably additive) if,

$$
\mu\left(\bigcup_{k=1}^{\infty} A_{k}\right)=\sum_{k=1}^{\infty} \mu\left(A_{k}\right)
$$

, whenever $\left\{A_{n}\right\}$ is a sequence of disjoint sets in $\mathcal{F}$

(8) Measure, if $\mu$ is $\sigma$-additive and $\mu(A) \geq 0$ for all $A \in \mathcal{F}$.

(9) Probability, if $\mu$ is a measure and $\mu(\Omega)=1$.

(10) Continuous from below at $A \in \mathcal{F}$, if $\lim _{n \rightarrow \infty} \boldsymbol{\mu}\left(\boldsymbol{A}_{n}\right)=\boldsymbol{\mu}(\boldsymbol{A})$, whenever

$\left\{A_{n}\right\}$ is a sequence of sets in $\mathcal{F}$, and $A_{n} \uparrow A$.

(11) Continuous from above at $A \in \mathcal{F}$, if $\lim _{n \rightarrow \infty} \mu\left(A_{n}\right)=\boldsymbol{\mu}(\boldsymbol{A})$, whenever $\left\{A_{n}\right\}$ is a sequence of sets in $\mathcal{F}$, and $A_{n} \downarrow A$.

(12) Continuous at $A \in \mathcal{F}$, if it is continuous both from below and from above at $A$.

Definition (4): [4]

$\operatorname{Let}(\Omega, \mathcal{F})$ be a fuzzy measurable space. A set function $\mu: \mathcal{F} \rightarrow[0, \infty]$ is said to be a fuzzy measure on $(\Omega, \mathcal{F})$ if it satisfies the following properties:

(1) $\mu(\emptyset)=0$

(2) If $A, B \in \mathcal{F}$ and $A \subseteq B$, then $\quad \mu(A) \leq$ $\mu(B)$

Definition (5): [5]

Let $(\Omega, \mathcal{F})$ be a fuzzy measurable space. A set function $\mu: \mathcal{F} \rightarrow[0, \infty]$ is called:

(1) Upper semi continuous fuzzy measure if and only if

$$
\lim _{n \rightarrow \infty} \mu\left(\mathrm{A}_{\mathrm{n}}\right)=\mu\left(\bigcup_{n=1}^{\infty} \mathrm{A}_{n}\right)
$$

whenever $\left\{A_{n}\right\}$ is increasing sequence.
(2)

Lower semi continuous fuzzy measure if and only if

$$
\lim _{n \rightarrow \infty} \mu\left(\mathrm{A}_{\mathrm{n}}\right)=\mu\left(\bigcap_{n=1}^{\infty} \mathrm{A}_{n}\right)
$$

whenever $\left\{A_{n}\right\}$ is decreasing sequence.

(3) Semi continuous fuzzy measure if it is both upper and lower semi continuous fuzzy measure.

(4) Regular if and only if

\section{Definition (6): [5]}

$$
\Omega \in \mathcal{F} \text { and } \mu(\Omega)=1 .
$$

Let $(\Omega, \mathcal{F})$ be a fuzzy measurable space. A set function $\mu: \mathcal{F} \rightarrow[0, \infty)$ is said to be

1. Exhaustive if

$\mu\left(A_{n}\right) \rightarrow 0$ whenever $\left\{A_{n}\right\}$ is infinite sequence of disjoint sets in $\mathcal{F}$

2. Order-continuous if $\mu\left(A_{n}\right) \rightarrow 0$, whenever $A_{n} \in \mathcal{F}, n=1,2, \ldots$ and $A_{n} \downarrow \emptyset$.

Definition (7): [6]

Let $(\Omega, \mathcal{F})$ be a fuzzy measurable space. A set function $\mu: \mathcal{F} \rightarrow[0, \infty)$ is said to be additive, if $\mu(A \cup B)=\mu(A)+\mu(B)$ whenever $A, B \in \mathcal{F}$ and $A \cap B=\emptyset$.

\section{Definition (8): $[6,7]$}

Let $(\Omega, \mathcal{F})$ be a fuzzy measurable space. A set function $\mu: \mathcal{F} \rightarrow[0, \infty)$ is said to be

Null-additive, if $\mu(A \cup B)=\mu(A) \quad$ whenever $A, B \in \mathcal{F}$ such that $A \cap B=\emptyset$, and $\mu(B)=$ 0 .

\section{Definition (9): [8]}

Let $(\Omega, \mathcal{F})$ be a fuzzy measurable space. A set function $\mu: \mathcal{F} \rightarrow[0, \infty)$ is said to be weakly null-additive, if for any $A, B \in \mathcal{F}$,

\section{Remark (10):}

$$
\mu(A)=\mu(B)=0 \Rightarrow \mu(A \cup B)=0
$$

The concept of null-null additive stems from a wings textbook which the book[8] derived from, in which it is said to be weak null additive. But we consider that it is more precise and vivid to call it "null- null additive".

\section{Definition (11):}

Let $(\Omega, \mathcal{F})$ be a fuzzy measurable space. A set function $\mu: \mathcal{F} \rightarrow[0, \infty)$ is said to be

finitely weakly null-additive, if for any $\left\{\mathrm{A}_{\mathrm{i}}\right\} \subset \mathcal{F}, \mu\left(A_{i}\right)=0$

$$
\text { , for all } \mathrm{i}=1, \ldots \ldots, \mathrm{n} \Rightarrow \mu\left(\bigcup_{i=1}^{n} A_{i}\right)=0
$$




\section{Definition (12): [6]}

Let $(\Omega, \mathcal{F})$ be a fuzzy measurable space. A set function $\mu: \mathcal{F} \rightarrow[0, \infty)$ is said to be

Countably weakly null-additive, if for any $\left\{\mathrm{A}_{\mathrm{n}}\right\} \subset \mathcal{F}, \mu\left(A_{n}\right)=0$

$$
\text { , for all } \mathrm{n} \geq 1 \Rightarrow \mu\left(\bigcup_{n=1}^{\infty} A_{n}\right)=0
$$

Definition (13): [6]

Let $(\Omega, \mathcal{F})$ be a fuzzy measurable space. A set function $\mu: \mathcal{F} \rightarrow[0, \infty)$ is said to be null-continuous, if $\mu\left(\bigcup_{n=1}^{\infty} A_{n}\right)=0$ for every increasing sequence $\left\{\mathrm{A}_{\mathrm{n}}\right\}$ in $\mathcal{F}$ such that $\mu\left(A_{n}\right)=0$, for all $n \geq 1$.

\section{Definition (14):[9]}

Let $(\Omega, \mathcal{F})$ be a fuzzy measurable space. A set function $\mu: \mathcal{F} \rightarrow[0, \infty)$ is said to be null-subtractive, if we have

$\mu\left(A \cap B^{c}\right)=\mu(A)$, whenever $A, B \in \mathcal{F}$ and $\mu(B)=0$.

\section{Definition (15):[9]}

Let $A \in \mathcal{F}, \mu(A)<\infty$. $\mu$ is called pseudo-nullsubtractive with respect to $A$, if for any $B \in A \cap \mathcal{F}$, we have

$\mu(B \cap C)=\mu(B)$, whenever $C \in \mathcal{F}, \quad \mu(A \cap$ $C)=\mu(A)$.here

$A \cap \mathcal{F}=\{A \cap D: D \in \mathcal{F}\}$.

\section{Definition (16):[9]}

Let $(\Omega, \mathcal{F})$ be a fuzzy measurable space. A set function $\mu: \mathcal{F} \rightarrow[0, \infty)$ is said to be

auto continuous from above (resp. autocontinuous from below), if $\mu\left(B_{n}\right) \rightarrow 0$ implies $\mu\left(A \cup B_{n}\right) \rightarrow \mu(A)$ (resp.

$\left.\mu\left(A \cap B_{n}^{c}\right) \rightarrow \mu(A)\right)$

, whenever $A \in \mathcal{F},\left\{B_{n}\right\} \subset \mathcal{F}, \quad \mu$ is called autocontinuous if it is both autocontinuous from above and autocontinuous from below.

Definition (17): [9]

Let $A \in \mathcal{F}, \mu(A)<\infty, \mu$ is called pseudoautocontinuous from above with respect to $A$ (resp. from below with respect to $A$ ), if for any $\left\{B_{n}\right\} \subset \mathcal{F}$, when

$\mu\left(B_{n} \cap A\right) \rightarrow \mu(A)$, then

$\left.\mu\left(B_{n}^{c} \cap A\right) \cup C\right) \rightarrow \mu(C)$,

(resp. $\mu\left(B_{n} \cap C\right) \rightarrow \mu(C)$ whenever

$C \in A \cap \mathcal{F}$.

$\mu$ is called pseudo-autocontinuous with respect to $A$,if it is both pseudo-autocontinuous from above with respect to Aand pseudoautocontinuous from below with respect to $A$.

\section{Main results}

Theorem (1):

Let $(\Omega, \mathcal{F}, \mu)$ be a fuzzy measure space, if $\mu$ is $\sigma-$ additive then

$\mathcal{F}^{*}=\{A \Delta B, A \in \mathcal{F}, B \subseteq \Omega$ and $\mu(B)=0\} \quad$ is fuzzy $\sigma$-field on $\Omega$.
Proof:

(1) Since $\Omega \Delta \emptyset=\Omega, \Omega \in \mathcal{F}$ ,$\emptyset \subseteq \Omega$ and $\mu(\emptyset)=0$

, we have $\Omega \in \mathcal{F}^{*}$

(2) Let $V \in \mathcal{F}^{*}$, we have $V=A \Delta B, A \in \mathcal{F}, B \subseteq \Omega$ with $\mu(B)=0$

$\Rightarrow V^{c}=(A \Delta B)^{c}=[(A / B) \cup(B / A)]^{c}$

$$
=\left(A^{c} \cap B^{c}\right) \cup(A \cap B)
$$$$
=\left(A^{c} / B\right) \cup\left(B / A^{c}\right)
$$

$=A^{c} \Delta B$, where $B \subseteq \Omega$ with $\mu(B)=0$

Since $A \in \mathcal{F}$ and $\mathcal{F}$ is a fuzzy $\sigma$-field, we have

$A^{c} \in \mathcal{F}$ and $B \subseteq \Omega$ with $\mu(B)=0$

$\Longrightarrow V^{c}=A^{c} \Delta B, A^{c} \in \mathcal{F}$ and

$B \subseteq \Omega$ with $\mu(B)=0$

$$
\therefore V^{c} \in \mathcal{F}^{*}
$$

(3) Let $\left\{V_{n}\right\}$ be a sequence of sets in $\mathcal{F}^{*}$ with

$V_{n}=A_{n} \Delta B_{n}, A_{n} \in \mathcal{F}, B_{n} \subseteq \Omega$ and $\mu\left(B_{n}\right)=0$ for all $n$.

We have

$$
\bigcup_{n=1}^{\infty} A_{n} \in \mathcal{F}
$$

$\mathcal{F}$ is fuzzy $\sigma-$ field

$\bigcup_{n=1}^{\infty} B_{n} \subseteq \Omega$ and $\mu\left(\bigcup_{n=1}^{\infty} B_{n}\right)=\sum_{n=1}^{\infty} \mu\left(B_{n}\right)=\mathbf{0}$

So

$$
\begin{aligned}
\bigcup_{n=1}^{\infty} V_{n}=\bigcup_{n=1}^{\infty}( & \left.A_{n} \Delta B_{n}\right) \\
& =\bigcup_{n=1}^{\infty}\left[\left(A_{n} / B_{n}\right)\right. \\
& \left.\cup\left(B_{n} / A_{n}\right)\right] \\
& =\bigcup_{n=1}^{\infty} A_{n} \Delta \bigcup_{n=1}^{\infty} B_{n} \\
\Rightarrow \bigcup_{n=1}^{\infty} V_{n} \in \mathcal{F}^{*} &
\end{aligned}
$$

Consequently $\mathcal{F}^{*}$ is fuzzy $\sigma$-field on $\Omega$.

\section{Remark (2):}

The union of a collection of fuzzy $\sigma$-field need not be fuzzy $\sigma$-field as in the following example.

Example (3):

Let $A, B, C, D$ are fuzzy sets and $\Omega=$ $\{A(x), B(x), C(x), D(x)\}$, such that

$A(x)= \begin{cases}2 x & 0 \leq x \leq 1 / 2 \\ 0 & 1 / 2<x \leq 1\end{cases}$

$B(x)=\left\{\begin{array}{lr}0 & 0 \leq x \leq 1 / 4 \\ 2 x & 1 / 4<x \leq 1 / 2 \\ 1 & 1 / 2<x \leq 1\end{array}\right.$

$C(x)= \begin{cases}1-2 x & 0 \leq x \leq 1 / 2 \\ 1 & 1 / 2<x \leq 1\end{cases}$ 


$$
D(x)=\left\{\begin{array}{lr}
1 & 0 \leq x \leq 1 / 4 \\
1-2 x & 1 / 4<x \leq 1 / 2 \\
0 & 1 / 2<x \leq 1
\end{array}\right.
$$

Let $\quad \mathcal{F}_{1}=\{\varnothing, A(x), C(x), \Omega\} \quad, \quad \mathcal{F}_{2}=$ $\{\varnothing, B(x), D(x), \Omega\}$ are two fuzzy $\sigma$-fields, but $\mathcal{F}_{1} \cup \mathcal{F}_{2}$ is not fuzzy $\sigma$-field.

Solution:

First we must prove that $\mathcal{F}_{1}$ and $\mathcal{F}_{2}$ is fuzzy $\sigma$-field.

$\mathcal{F}_{1}$ Is fuzzy $\sigma$-field

(1) $\emptyset, \Omega \in \mathcal{F}_{1}$.

(2) (i) Let $A(x) \in \mathcal{F}_{1}$, to prove $A^{c}(x) \in \mathcal{F}_{1}$

From Definition (1.1.6) we get on

$$
\begin{gathered}
=1- \begin{cases}2 x & A^{c}(x)=1-A(x) \\
0 & 0 \leq x \leq 1 / 2\end{cases} \\
= \begin{cases}1-2 x & 0 \leq x \leq 1 / 2 \\
1 & 1 / 2<x \leq 1\end{cases} \\
=C(x)
\end{gathered}
$$

But $C(x) \in \mathcal{F}_{1}$ $\Rightarrow A^{c}(x) \in \mathcal{F}_{1}$.

(ii) Let $C(x) \in \mathcal{F}_{1}$, to prove $C^{c}(x) \in \mathcal{F}_{1}$ $C^{c}(x)=1-C(x)$

$$
\begin{aligned}
& =1 \\
& - \begin{cases}1-2 x & 0 \leq x \leq 1 / 2 \\
1 & 1 / 2<x \leq 1\end{cases} \\
& = \begin{cases}2 x & 0 \leq x \leq 1 / 2 \\
0 & 1 / 2<x \leq 1\end{cases} \\
& =A(x)
\end{aligned}
$$

But $A(x) \in \mathcal{F}_{1}$

$$
\Rightarrow C^{c}(x) \in \mathcal{F}_{1}
$$

(iii) It is clear that $\emptyset^{c}=\Omega \in \mathcal{F}_{1}$

And $\Omega^{c}=\emptyset \in \mathcal{F}_{1}$

$$
\text { (3) (i) if } 0 \leq x \leq 1 / 2
$$

$$
\begin{aligned}
\Rightarrow(A \cup C)(x)= & \max \{A(x), C(x)\} \\
= & \max \{2 x, 1-2 x\}=2 x \\
& \text { If } x=0 \\
& \Rightarrow(A \cup C)(x)=0 \\
& =\emptyset(x) \in \mathcal{F}_{1} .
\end{aligned}
$$

$$
\begin{aligned}
& (A \cup C)(x)=1= \\
& \Omega(x) \in \mathcal{F}_{1} .
\end{aligned}
$$

(ii) $1 / 2<x \leq 1$

$$
\begin{aligned}
\Rightarrow(A \cup C)(x)= & \max \{A(x), C(x)\} \\
& =\max \{0,1\}=1
\end{aligned}
$$

$\therefore A \cup C(x)=1=\Omega(x) \in \mathcal{F}_{1}$.

$\therefore \mathcal{F}_{1}$ Is fuzzy $\sigma$-field

In the same way we can prove that $\mathcal{F}_{2}$ is fuzzy $\sigma$-field.

Now to prove that $\mathcal{F}_{1} \cup \mathcal{F}_{2}$ is not fuzzy $\sigma$-field

$\rightarrow \mathcal{F}_{1} \cup \mathcal{F}_{2}=\{\varnothing, A(x), B(x), C(x), D(x), \Omega\}$

$$
A(x)=\left\{\begin{array}{lr}
2 x & 0 \leq x \leq 1 / 4 \\
2 x & 1 / 4<x \leq 1 / 2 \\
0 & 1 / 2<x \leq 1
\end{array}\right.
$$

$\Rightarrow(A \cup B)(x)=\max \{A(x), B(x)\}=$ $\max \{2 x, 0\}=2 x$

$$
\begin{array}{ll}
\text { (a) } & \text { If } \quad x=0 \Rightarrow A \cup \\
& B(x)=0=\varnothing(x) \in \\
& \mathcal{F}_{1} . \\
\text { (b) } \quad x=\frac{1}{4} \Rightarrow(A \cup \\
\text { If } \quad B)(x)=1 / 2 \notin \mathcal{F}_{1} .
\end{array}
$$

$\therefore \mathcal{F}_{1} \cup \mathcal{F}_{2}$ is not fuzzy $\sigma-$ field

\section{Theorem (4):}

Let $(\Omega, \mathcal{F}, \mu)$ be a fuzzy measure space, suppose that $\mathcal{F}^{*}$ is $\sigma$-field and $\mu^{*}$ is a measure on $\left(\Omega, \mathcal{F}^{*}\right)$, for any $A \in \mathcal{F}$ such that $\mu(B)=\mu^{*}(A \cap B)$ For any $B \in \mathcal{F}^{*}$ is fuzzy measure on $(\Omega, \mathcal{F})$.

\section{Proof:}

(1) Since $\mathcal{F}^{*}$ is $\sigma$-field $\Longrightarrow \emptyset \in \mathcal{F}^{*}$

$\therefore \mu(\varnothing)=\mu^{*}(A \cap \emptyset)=\mu^{*}(\varnothing)=\varnothing$.

(2) Let $A_{1}, A_{2} \in \mathcal{F}$, if $A_{1} \subseteq A_{2}$, then $\mu\left(A_{1}\right)=\mu^{*}\left(A_{1} \cap B\right) \leq \mu^{*}\left(A_{2} \cap B\right)=\mu\left(A_{2}\right)$

$\therefore \mu$ is fuzzy measure on $(\Omega, \mathcal{F})$.

Theorem (5):

Let $(\Omega, \mathcal{F}, \mu)$ be a fuzzy measure space such that there is $B \in \mathcal{F}$ with

$0<\mu(B)<\infty$, define $\mu^{*}: \mathcal{F} \rightarrow[0, \infty]$ by $\mu^{*}(A)=\mu(A \cap B) / \mu(B), \operatorname{then}\left(\Omega, \mathcal{F}, \mu^{*}\right) \quad$ is fuzzy measure space.

\section{Proof:}

$\mu^{*}(\varnothing)=\mu(\varnothing \cap B) / \mu(B)=0$.

let $A, B \in \mathcal{F}$, if $A \subseteq B$, we have $\mu(A) \leq$ $\mu(B)$

Since $A \subseteq B$, hence $A \cap B=A$

$$
\begin{gathered}
\Rightarrow \mu(A \cap B)=\mu(A) \\
\Rightarrow \mu(A \cap B)=\mu(A) \leq \mu(B) \\
\Rightarrow \mu(A \cap B) / \mu(B) \leq \mu(B \cap B) / \mu(B) \\
\Rightarrow \mu^{*}(A) \leq \mu^{*}(B) .
\end{gathered}
$$

Consequently $\mu^{*}$ is a fuzzy measure.

Theorem (6):

Let $(\Omega, \mathcal{F})$ be a fuzzy measurable space, $\mu, v$ be a fuzzy measures on $\Omega$, then $\mu+v$ which denoted by

is fuzzy measure on $\Omega$.

$$
(\mu+v)(A)=\mu(A)+v(A)
$$

\section{Proof:}

(1) Since $\mu, v$ be two fuzzy measures

$\Longrightarrow(\mu+v)(\varnothing)=0$.

(2) let $A, B \in \mathcal{F}$, if $A \subseteq B$, we have

$(\mu+v)(A)=\mu(A)+v(A) \leq \mu(B)+v(B)$

$=(\mu+v)(B)$.

So $\mu+v$ is fuzzy measure. 


\section{Corollary (1):}

Let $(\Omega, \mathcal{F})$ be a fuzzy measurable space, $\mu$ be a fuzzy measure on $\Omega$, and $\alpha>0$, define a set function $(\alpha \mu)(A)=\alpha \mu(A)$, then $\alpha \mu$ is fuzzy measure on $\Omega$.

Proof:

(1) Since $\mu$ be a fuzzy measure, we have $(\alpha \mu)(\varnothing)=\alpha \mu(\emptyset)=0$.

(2) let $A, B \in \mathcal{F}$, if $A \subseteq B$, we have

$$
\begin{gathered}
\mu(A) \leq \mu(B) \\
\Rightarrow(\alpha \mu)(A)=\alpha \mu(A) \leq \alpha \mu(B) \\
=(\alpha \mu)(B) .
\end{gathered}
$$

So $\alpha \mu$ is fuzzy measure.

Remark (7)

The points (1) and (2) from Definition (5) explain fuzzy measure is upper semi continuous and lower semi continuous; the following results take us to the converse direction.

\section{Theorem (8)}

Let $(\Omega, \mathcal{F})$ be a fuzzy measurable space and let $\mu$ be a function $\mu: \mathcal{F} \rightarrow \mathbb{R}_{+}$, if $\mu$ is additive, non-decreasing and upper semi continuous, then $\mu$ is fuzzy measure.

Proof:

$$
\text { (1) Since } A=A \cup \emptyset
$$

Also $\mu$ is additive we have

$$
\begin{gathered}
\mu(A)=\mu(A \cup \emptyset)=\mu(A)+\mu(\emptyset) \\
\Rightarrow \mu(\emptyset)=0
\end{gathered}
$$

(1) Let $A, B \in \mu$, if $A \subseteq B$, we have $\mathrm{B}=A \cup(B \backslash A)$

$$
\text { and } A \cap(B \backslash A)=\emptyset
$$

Since $\mu$ is additive we have, we obtain

Consequently

$$
\mu(B)=\mu(A)+\mu(B \backslash A)
$$

$$
\mu(B \backslash A)=\mu(B)-\mu(A)
$$

In addition, $\mu(B \backslash A) \geq 0$

Hence

Then $\mu$ is fuzzy measure.

$$
\mu(A) \leq \mu(B)
$$

\section{Theorem (9):}

Let $(\Omega, \mathcal{F})$ be a fuzzy measurable space , let $\left\{\mathrm{A}_{n}\right\}$ be a sequence of disjoint fuzzy set in $\mathcal{F}$ and it is decreasing, if $\mu\left(\mathrm{A}_{n}\right)<\infty$ and $\mu$ is lower semi continuous fuzzy measure at $\emptyset$, then $\lim _{n \rightarrow \infty} \mu\left(\mathrm{A}_{n}\right)=0$.

Proof:

Since $\left\{A_{n}\right\}$ is lower continuous fuzzy measure at $\varnothing$, we have

But

$$
\lim _{n \rightarrow \infty} \mu\left(A_{n}\right)=\mu(\varnothing)
$$

Consequently, we have

$$
\mu(\varnothing)=0
$$

\section{Theorem (10):}

$$
\lim _{n \rightarrow \infty} \mu\left(\mathrm{A}_{n}\right)=0 \text {. }
$$

Let $(\Omega, \mathcal{F})$ be a fuzzy measurable space, and for any $A \in \mathcal{F}, \quad \mu(A) \neq 0$, then $\mu$ is null additive.

\section{Proof:}

If there exists some set $B \in \mathcal{F}$ such that $\mu(B)=0$, then $B=\emptyset$.

Consequently, for any $A \in \mathcal{F}$, we have $\mu(A \cup B)=\mu(A)$.

\section{Theorem (11):}

Let $(\Omega, \mathcal{F})$ be a fuzzy measurable space, if $\mu$ is autocontinuous from below, then it is nullsubtractive.

\section{Proof:}

Let $A, B_{n} \in \mathcal{F}$

Since if $\mu$ is autocontinuous from below, we have

Also we have

$$
\lim _{n \rightarrow \infty} \mu\left(B_{n}\right)=0
$$

$$
\mu\left(A \cap B_{n}^{c}\right) \rightarrow \mu(A)
$$

Consequently $\mu$ is null-subtractive.

\section{Theorem (12):}

Let $(\Omega, \mathcal{F})$ be a fuzzy measurable space, if $\mu$ is pseudo-autocontinuous from below with respect to $A$, then it is pseudo-nullsubtractice with respect to $A$.

\section{Proof:}

$$
\text { Let } A, B_{n} \in \mathcal{F}
$$

Since if $\mu$ is pseudo-autocontinuous from below, we have

$\mu(A)<\infty$. And $C \in A \cap \mathcal{F}$

$$
\mu\left(B_{n} \cap C\right) \rightarrow \mu(C)
$$

Consequently $\mu$ is pseudo-null-subtractice with respect to $A$.

Theorem (13):

Let $(\Omega, \mathcal{F})$ be a fuzzy measurable space, if $\mu$ is upper semi continuous fuzzy measure and ccountably weakly null additive then $\mu$ is exhaustive.

Proof:

Let $\left\{A_{n}\right\}$ be a disjoint of sequence of sets in $\mathcal{F}$ Since $\mu$ is countably weakly null additive $\therefore \mu\left(A_{n}\right)=0$, for all $n \geq 1$

$$
\Rightarrow \mu\left(\bigcup_{n=1}^{\infty} A_{n}\right)=0
$$

Also $\mu$ is upper semi continuous

$$
\therefore \mu \text { is exhaustive. }
$$

$$
\begin{gathered}
\Rightarrow \lim _{n \rightarrow \infty} \mu\left(A_{n}\right)=\mu\left(\bigcup_{n=1}^{\infty} A_{n}\right) \\
\Rightarrow \lim _{n \rightarrow \infty} \mu\left(A_{n}\right)=0
\end{gathered}
$$

\section{Theorem (14):}

Let $(\Omega, \mathcal{F})$ be a fuzzy measurable space, if $\mu$ is ccountably weakly null additive then $\mu$ is nullcontinuous. 


\section{Proof:}

Let $\left\{A_{n}\right\}$ be a increasing sequence of sets in $\mathcal{F}$, such that

$$
\mu\left(A_{n}\right)=0 \text {, for all } n \geq 1
$$

Since $\mu$ is ccountably weakly null additive

$$
\Rightarrow \mu\left(\bigcup_{n=1}^{\infty} A_{n}\right)=0
$$

$\therefore \mu$ is null-continuous .

\section{References}

[1] Qiao Zhong,"Riesz's theorem and Lebesgue's theorem on the fuzzy measure space", busefal 29, (1987), 33-41.

[2] E. P. Klement, "Fuzzy u-algebras and fuzzy measurable functions", Fuzzy Sets and Systems 4, (1980), 83-93.

[3] Ash, R.B, "Probability and Measure Theory" Second edition, 2000, London.

[4] Sugeno, .M" Theory of Fuzzy Integrals and Its Applications", Ph.D. Dissertation, Tokyo Institute of Technology, 1975.

[5] G. J. Klir, "Convergence of sequences of measurable functions on fuzzy measure space ", fuzzy set and system 87, (1997) ,317-323.

[6] Jun Li, Radko Mesiar and Endre Pap, "Atoms of weakly null- additive monotone measures and integrals", Information Science 257, (2014), 183-192.

[7] Wang Zhenyuan, "The Autocontinuity of Set Function and the Fuzzy Integral", journal of mathematical analysis and application, 99, (1984), 195-218.

[8] Z. Wang and G. J. Klir, "Fuzzy measure theory" Plenum Press, New York, 1992.
[9] QIAO Zhong," On Fuzzy Measure and Fuzzy Integral on Fuzzy Set", Fuzzy Sets and Systems 37,(1990),77-92 North -Holland.

[10] D.Ralescu, G.Adms, "The fuzzy integral",J.Math.Anal.Appl.75,(1980),562-570. [11] Wang Zhenyuan, "Asymptotic structural characteristics of fuzzy measure and their applications", Fuzzy Sets and Systems 16, (198 5), 277- 290.

[12] Kruse, R., "on the construction of fuzzy measures", Fuzzy Sets and Systems, 8, (1982), 323-327.

[13] L. Y. Kui, "The completion of fuzzy measure and its applications", Fuzzy sets and Systems 146, (2001), 137-145.

[14] Q. Jiang, H. Suzuki, "Lebesgue and Saks decompositions of $\sigma$-finite fuzzy measure", Fuzzy Set and Systems, 75, (1995), 181-201.

[15] L. Lushu and S. Zhaohu, "The fuzzy setvalued measures generated by fuzzy random variables", Fuzzy Set and Systems, 97, (1998), 203-209.

[16] H. Minghu, W. Xizhao and W. Congxin,"Fundamental convergence of sequence of measureable functions on fuzzy measure space", Fuzzy Sets and Systems, 95,(1998), 77-81.

[17] Kruse, R., "on the construction of fuzzy measures", Fuzzy Sets and Systems, 8, (1982), 323-327.

[18] L. A. Zadeh, Fuzzy sets, Information and Control, 8, (1965), 338-353.

[19] H. J. Zimmerman, "fuzzy set theory and Its Application", Kluwer Academic Publisher, 1991.

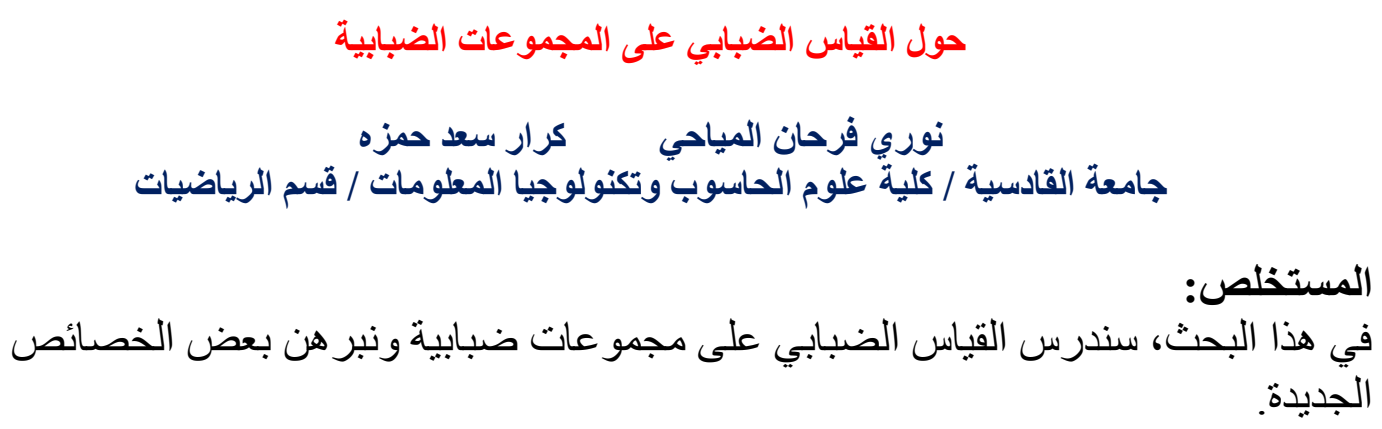

